\title{
Post-Traumatic Epilepsy: Review
}

\section{Edward Cesnik, llaria Casetta, Enrico Granieri*}

Department of Medical and Surgical Sciences of Communication and Behaviour, University of Ferrara, Italy

\begin{abstract}
Post traumatic epilepsy is defined by recurrent seizures secondary to brain injury following head trauma and represent the most frequent cause of epilepsy in young adults. Most post traumatic epilepsies start within two years from trauma, but in some cases may present several years after the brain injury. Severe traumatic brain injuries tend to correlate with an increased risk of developing early and late post-traumatic seizures. A cranial trauma creates a potentially epileptogenic brain damage through a number of different mechanisms. Several structural, physiological and biochemical modifications occur in a brain after a head injury that promote oxidative stress mechanisms and excitotoxic mechanisms. Current evidence shows that prophylactic use of antiepileptic drugs prevent the occurrence of early post-traumatic seizure but does not influence the incidence of post-traumatic epilepsy. Some authors propose treatment with antioxidant drugs in acute phase of severe traumatic brain injuries in order to prevent early tissue changes established in the traumatized tissue.
\end{abstract}

Keywords: Post-traumatic epilepsy; Post-traumatic seizures; Traumatic brain injuries; Head trauma

\section{Introduction}

Post-traumatic epilepsy (PTE) is a form of symptomatic epilepsy defined by the presence of recurrent seizures secondary to traumatic brain injury [1]. It is presumed that there is a mechanism induced by a head trauma that leads the brain tissue to develop a chronic predisposition to the development of seizures. We distinguish from immediate post-traumatic seizures (PTSs) which occur within 24 hours after injury, early seizures which occur within a week after injury and finally late seizures which occur after one week after injury. Immediate and early seizures belong to the group of provoked seizures and they do not define epilepsy since they are not underlying a pathogenic mechanism that chronically predisposes the patient to manifest epileptic seizures [2]. Post-traumatic epilepsy is determined by the presence of late seizures and therefore unprovoked.

\section{Epidemiology}

Post-traumatic epilepsy (PTE) is the most frequent cause of epilepsy in young adults as they are more at risk of the exposure to head injury [3,4]. Traumatic head injury (TBI) represent one of the most important epilepsy risk factors in children, with increased epilepsy risk 7.02-fold (for severe TBI) in univariate analysis [5]. Estimated proportion of presumed causes of incident epilepsy related to TBI range between $2 \%$ and $16 \%$ in European Countries [6]. The study of PTE epidemiology stems from the analysis of military records at the beginning of the twentieth century, with reference to the American Civil War, to the First World War, to the Second World War and other more recent studies have been carried out on traumatized soldiers from the Korean War and the War in Vietnam [7-11]. PTE epidemiology mainly uses frequency rates of epilepsy in traumatized patients and rates of "incidence ratio" between population with head injury and general population. Severe traumatic brain injuries tend to correlate with an increased risk of developing early and late post-traumatic seizures compared to mild and moderate TBIs [12]. Obtaining reliable epidemiological data on the frequency of epilepsy in patients with TBI requires prospective studies with long periods of follow-up (at least 10 years). Although, at a distance of 10-15 years, it is difficult to establish, with certainty, a direct cause-effect correlation between head trauma and incident epilepsy In industrialized countries the proportion of epilepsy attributed to brain injuries is higher in past studies than in recent ones [4,13]. TBIs and other risk factors have actually modified their frequency over time; the reasons are attributable to modification in socio-economic status and amelioration in emergency health care. However, improved survivorship of severe traumatized patients could increase proportion of PTE in future epidemiological investigations.

\section{Early and immediate PTSs}

Early PTSs occur in various series of cases with a variable frequency comprised between $2.6 \%$ and $16.9 \%$, depending on the severity of TBI $[14,15]$. In the infant population early epileptic seizures are much more frequent compared to late seizures and they very often tend to occur as immediate seizures (within 24h) [16]. In general, severe TBIs are associated with a high frequency of early crises, with rates that in adults are 20-30 times higher in comparison with mild and moderate TBIs [17].

\section{Late PTSs}

Frequency rates of late PTSs are variable in different studies with values comprised between $1.9 \%$ and $30 \%$; such differences are correlated to the different entity of TBIs in the various series of cases. Annegers et al. have published a prospective study with a follow-up of over 10 years, reporting an average PTE frequency of $2.1 \%$ in TBI patients; the percentage was significantly higher $(12 \%)$ if considering only subjects with severe TBI [14]. PTE frequency and incidence studies in military populations inevitably show higher rates compared to studies carried out in the general population, with regard to a higher risk of severe TBIs. In fact, military series of cases show frequency rates varying from 34\% [10] to 53\% [11]. Most patients with PTE tend to have the first unprovoked seizures (PTSs) within the first year from trauma (approximately $80 \%$ ) and in almost all of them they occur within the

*Corresponding author: Enrico Granieri, Neurological Clinic, Department of Medical and Surgical Sciences of Communication and Behaviour, University of Ferrara, Italy, Tel: 390532 236633; Fax: 390532 239649; E-mail: gnr@unife.it

Received May 27, 2013; Accepted June 20, 2013; Published June 25, 2013

Citation: Cesnik E, Casetta I, Granieri E (2013) Post-Traumatic Epilepsy: Review. J Neurol Neurophysiol S2: 009 doi:10.4172/2155-9562.S2-009

Copyright: $\odot 2013$ Cesnik E, et al. This is an open-access article distributed under the terms of the Creative Commons Attribution License, which permits unrestricted use, distribution, and reproduction in any medium, provided the original author and source are credited. 
second year. Annegers calculated that a patient with TBI has a risk of unprovoked seizures during the first year ("incidence ratio") eleven times higher than the general population, 3.5 times in the following 4 years, 2.39 times after 5 years and 1.56 times 10 years after injury. A patient with mild TBI shows a normalization of the risk (incidence ratio 1.1) after the first 5 years, while a patient with severe TBI shows high values even after 10 years (incidence ratio 4.0) [14].

\section{Risk Factors}

Epidemiological studies have generally revealed a higher risk of early and late seizures in relation to the severity of the head injury [18]. The characteristics of the trauma and the presence of any associated lesions represent risk factors for a successive development of early and immediate PTSs or late PTSs. A recent study [19] that retrospectively compared a population of subjects without head injury (non TBI group) and subjects with moderate and severe TBI with skull fracture revealed, on behalf of the latter, a relative risk of $10.6 \%$ (HRs) to develop epilepsy. In the past, Jennet [20] showed that the presence of compound depressed skull fractures was associated to a higher general risk of early and late PTSs. The studies found the following risk factors for early epileptic seizures: acute subdural hemorrhage, acute intraparenchymal hemorrhage, need of surgery, infant age and amnesia lasting more than 30 minutes, history of chronic alcoholism. Risk factors for late PTSs are acute subdural hematomas, acute subdural hemorrhage, multiple brain contusions, age over 35, transient amnesia which lasts more than 24 hours and male gender [19]. Some studies have considered the presence of early PTSs as a risk factor for late PTSs in adults [20,21], but this is certainly not true for the infant population [20] and according to Annegers [14], it is not even a risk factor for the adult population. Acute subdural hematoma is the intracranial lesion which has highest risks of developing both early and late PTIs. The role of genetic susceptibility that regulate response to cerebral injury is not clear; most of epidemiological studies did not found that family history of epilepsy could represent a significant risk factor for early and late PTSs [22].

\section{Clinical Presentation}

Patients with PTE in most cases have focal epilepsy or focal epilepsy with a secondary generalization. The clinical characteristics of the seizures depend on the location of the lesion and the precocity of the secondary generalization. In patients with a history of trauma in childhood (less than five years) can manifest mesial temporal lobe epilepsies, as a trauma in the temporal region at an early age can induce the appearance of mesial temporal sclerosis $[23,24]$. Status epilepticus (SE) at onset is not uncommon; Jennett calculated a frequency of $10 \%$ in patients with PTSs [20]. The infant population undoubtedly has a higher probability of SE compared to the adult one.

\section{Course (Natural History)}

Most PTEs start within two years from trauma (about 90\%) [25], the first 18 months after injury is the period at a higher risk to develop late PTSs. Once PTE is diagnosed, the remission rates of seizures vary between $25-40 \%$. Patients with high frequencies of PTSs during the first year after injury have a low probability of remission.

\section{Pathogenesis}

There are several structural, physiological and biochemical modifications occuring in a brain after a head injury. A cranial trauma creates a potentially epileptogenic brain damage through a number of different mechanisms, which are not yet fully elucidated. In fact, different pathogeneses recognize early seizures and late seizures. The studies in this regard made use of animal and in vitro models with PTE. In 1978, Willmore [26] used an animal model where iron (ferric chloride) was injected into the cortex of rats, with an evidence of epileptiform anomalies in EcoG recordings after convulsive epileptic seizures. Direct inoculations of hemoglobin into brain cortex were used in other animal models [27] recently; some authors studied a PTE model induced by lateral fluid percussion of the rat cortex, with the detection of an increased susceptibility to seizures at a distance of 30 days [28]. In vitro models, thin layers of hippocampus are used in order to evaluate the response of the micro cortexes correlated to mechanic injuries or any changes in the concentrations of neurotransmitters. Immediate and early seizures are considered direct reactions of a brain damage and they are correlated to an altered vessel regulation of the local cerebral blood flow, to an alteration of the hematic-encephalic barrier and to an increase of the intracranial pressure with focal or diffused presence of ischemic, hemorrhagic, inflammatory or necrotic damage [29]. The results of studies conducted on animal models of chronic epilepsy have suggested that late seizures are caused by two main pathogenic pathways: oxidative stress mechanisms, excitotoxic mechanisms - neuronal hyper excitability. The mechanism of oxidative stress implies a trauma accompanied by a leakage of red blood cells, with a consequent formation of free radicals mediated by the iron contained in hemoglobin; these free radicals react with the methylene groups in the double lipid layer of neuronal membranes with subsequent lipid peroxidation of the neuronal membranes and mitochondria and the alteration of the function of the sodium/ potassium pump ATPase activity. The result of this sequence of events is a reduction of the chronic convulsive threshold of a group of neuronal cells. The excitotoxic mechanism is explained by the extra cellular increase of excitatory amino acids immediately after injury, with increased levels of glutamate and aspartatic acid [30]. These traumatized cells tend to assume excitatory aminoacids more readily than controls and present increased expression of the sodium-coupled neutral amino acid transporters subtypes 1 (SNAT1) and subtypes 2 (SNAT 2) [31]. Traumatized in vitro cells tend to form axonal sprouting with a higher immunoreactivity to GAP 43 (Grown Associated Protein); these cells show an altered excitability, evidenced by the presence of synaptic potentials with prolonged post-synaptic components [32]. According to some authors [33], the impaired neuronal plasticity in injured brain areas can promote the appearance of focal dysplasia if TBI occurs soon after birth when the process of neuronal migration is not yet fully completed.

\section{Diagnosis}

In patients with seizures at a short distance from cranial trauma it is necessary to exclude other potential causes of provoked seizures. A trauma patient often presents conditions of metabolic and circulatory instability, with high probability of alteration in the biochemical parameters, such as hyponatremia, which may lower the epileptogenic threshold.

\section{Electroencephalogram (EEG)}

EEG in a patient with TBI is useful for the localization of the lesion focus and for the measurements of the extent of damage, but it is not able to define the probability to develop epilepsy; more than $20 \%$ of patients with PTE have a negative EEG during the first three months after injury [34]. Anomalies may be present, ranging from a simple slowdown in the base activity of slow lesional waves in a localized focus. In patients with immediate seizures, sequences of spikes inscribed on 
slow focal activity can be noted, prevalently in the temporal regions (most exposed to contusive lesions). In later stages EEGs can be useful in predicting a possible recurrence of seizures before the prophylactic therapy is suspended [35].

\section{Neuroimaging}

In emergency "settings", the frontline examination is a CT scan of the head, a quick investigation and possible in any condition of the patient. The CT immediately shows the presence or absence of intra- or extra-parenchyma hemorrhagic foci, which can lead to a possible choice of neurosurgical treatment. Brain MRI represents the study of choice in patients with late seizures since it is an investigation that allows a better anatomy definition of the brain and gives the exact outcome of any post-traumatic lesion. T2-weighted images and gradient echo sequences may well highlight the presence of hemosiderin deposits that have a potential epileptogenic role. Messori et al. [36], through the analysis of T2 images from more than 130 patients, showed how a precocious formation of a gliotic scar around a hemosiderin deposit reduces the risk of PTE.

\section{Prophylaxis}

The use of anticonvulsants in acute phase of injury is quite common in clinical practice. The manifestation of immediate or early seizures can affect the patient's prognosis since it may increase the cerebral perfusion pressure and the intracranial pressure. The prophylactic therapy of PTE is mainly intended to block or delay epileptogenic mechanisms established after TBI. In the studies of epilepsy, "kindled" animal models are used in the testing of antiepileptic drugs; the choice of animal and "in vitro" models specific for PTE allows the study of, not only anticonvulsive properties of drugs, but also potential antiperoxidant affects which contrast the pathogenic mechanism underlying PTE. Some authors proposed to apply the experience derived from new approaches to the treatment of acute stroke, where the concept "time is brain" is the most important thing; the possibility of preventing early tissue changes established in the traumatized tissue may be the basis for the actual prophylactic treatment of PTE.

\section{Antiepileptic drugs}

The use of antiepileptic drugs (AEDs) at an early stage in order to prevent PTE is rather controversial. Several AEDs have been tested in observation studies and randomized clinical trials (RCTs) as a prophylactic therapy for PTE. The results of RCTs seem to show a moderate effect only on early PTEs, not on the late ones. Phenytoin (PHT) has been the most studied drug in patients with TBE since 1940 [37]. Temkin demonstrated that an early administration of PHT prevented early PTSs in an excellent way, while it did not prevent the complete onset of late PTSs both during the first year of therapy and in the following years without therapy [38]. A meta-analysis of all the trials conducted on PHT showed similar results [39]. The administration of PHT with Phenobarbital (PB) had been studied in several series of studies, starting from 1979, without providing any univocal and significant data of effectiveness. There is not much literature about Carbamazepine (CBZ); only one true randomized clinical trial is reported [40] and this showed a good efficacy in the prevention of early PTSs, but not in the late ones. Similar results have been reported for VPA [41], however with less capacity to prevent early PTSs compared with PHT. As for more recent drugs, data are still poor, since it is necessary to perform long term prospective studies. Among these, a particular attention has been paid to Levetiracetam (LEV), a drug that, unlike other AEDs, shows an efficacy both in kindling animal models and in PTE animal models. An observational study on severe head injuries revealed a good capacity of LEV to prevent early PTEs, comparable to PHT, but with a greater tendency to persistence of epileptiform EEG abnormalities [42]. At present, a "safety" RCT is going on, which is mainly considering the tolerability of LEV in PTE. Preliminary data show a low rate of adverse events and suspension of the drug, and a good efficacy with a temporary $53 \%$ reduction of PTE risk, although the percentage is likely to decrease during the prolongation of the follow-up [43]. Zonisamide is an antiepileptic drug which has showed antioxidant efficacy through the detoxification of $\mathrm{NO}$ and $\mathrm{OH}^{-}$ions (by scavenging $\mathrm{NO}$ and $\mathrm{OH}$ ions) with a consequent stabilization of the neuronal membrane [44]. In general, the recommendations for drug prophylaxis of early PTSs provide Phenytoin as frontline drug, Levetiracetam and Carbamazepine as second choice drugs; Phenobarbital and Valproate are not considered indicated for PTS prophylaxis. Drug prophylaxis of late PTSs is not recommended because of its absolute lack of evidence in observational clinical trials and RCT.

\section{Natural antioxidants}

The study of pathogenic mechanisms of PTE has been focusing on the use of antioxidant drugs in order to prevent the lipid peroxidation of neuronal membranes. In animal models, Tochopherol (vitamin E) has shown a reduction of electrical crises on EEGs, induced by the administration of $\mathrm{FeCl}_{2}$ (reduced onset of EEG seizures induced by intracerebral $\mathrm{FeC1}_{2}$ ) [45]. The combination of vitamin $\mathrm{E}$, ascorbic acid and vitamin $\mathrm{C}$ showed to have antiepileptic effects on the same animal models [46].

\section{Miscellaneous agents}

Magnesium sulfate was tested in the acute phase of injury for its neuroprotective capacity shown in studies in vitro; some observational studies and a RCT [47] were carried out and they revealed a reduction of early epileptic seizures, while late PTSs were unchanged. The statistical analysis of the trial results showed, however, very large confidence intervals, so large as not to consider the benefice statistically relevant on early PTSs. Adenosine showed anticonvulsant activity in several animal models of epilepsy through its action on A1 receptors [48]. The use of adenosine in animal models of PTE detected a detoxification capacity (scavenging) from free radicals released by the administration of $\mathrm{FeCl}_{2}$ [49]. Anticonvulsant properties of melatonin have been known for a long time; pinealectomia induces convulsive seizures in experimental animals [50]. Studies in vitro and above all on animal models have shown an ability of inhibition of the lipid peroxidation mechanism [51]. The pathogenic hypothesis of an excitotoxic mechanism in PTE focuses on medicines which can restrict the entry of intracellular calcium and antagonize NMDA receptors [52]. Calcium antagonists as Nimopidine have been used for several years in vasospasm prophylaxis in subarachnoid hemorrhage following a trauma or not, presenting a potential antiepileptogenic mechanism. Glutamate antagonists have been used in patients with ischemic stroke and TBI in several clinical trials, but without any significant results [53].

\section{Acknowledgment}

The authors thanks Dr. Eva Sjolin for her help in revising the translation of the manuscript.

\section{References}

1. Wrightson P, Gronwall D (1999) Mild head injury: Post-traumatic epilepsy Oxford University Press, London: 72-75. 
2. [No authors listed] (1993) Guidelines for epidemiologic studies on epilepsy. Commission on Epidemiology and Prognosis, International League Against Epilepsy. Epilepsia 34: 592-596

3. Hauser WA, Annegers JF, Kurland LT (1991) Prevalence of epilepsy in Rochester, Minnesota: 1940-1980. Epilepsia 32: 429-445

4. Granieri E, Rosati G, Tola R, Pavoni M, Paolino E, et al. (1983) A descriptive study of epilepsy in the district of Copparo, Italy, 1964-1978. Epilepsia 24: 502514

5. Cansu A, Serdaroğlu A, Yüksel D, Doğan V, Ozkan S, et al. (2007) Prevalence of some risk factors in children with epilepsy compared to their controls. Seizure 16: 338-344.

6. Forsgren L, Beghi E, Oun A, Sillanpää M (2005) The epidemiology of epilepsy in Europe - a systematic review. Eur J Neurol 12: 245-253.

7. Allen DP, Sanford HL, Dolley DH (1906) Traumatic defects of the skull: their relation to epilepsy: a clinical and experimental study of their repair. Boston Med Surg Journ 154: 396-405.

8. Ascroft PB (1941) Traumatic Epilepsy After Gunshot Wounds of the Head. Br Med J 1: 739-744.

9. Russell WR, Whitty CW (1952) Studies in traumatic epilepsy. I. Factors influencing the incidence of epilepsy after brain wounds. J Neurol Neurosurg Psychiatry 15: 93-98.

10. Caveness WF, Walker AE, Ascroft PB (1962) Incidence of posttraumatic epilepsy in Korean veterans as compared with those from World War I and World War II. J Neurosurg 19: 122-129.

11. Salazar AM, Jabbari B, Vance SC, Grafman J, Amin D, et al. (1985) Epilepsy after penetrating head injury. I. Clinical correlates: a report of the Vietnam Head Injury Study. Neurology 35: 1406-1414.

12. Frey LC (2003) Epidemiology of posttraumatic epilepsy: a critical review. Epilepsia 44 Suppl 10: 11-17.

13. Cesnik E, Pedelini F, Faggioli R, Monetti VC, Granieri E, et al. (2013) Incidence of epilepsy in Ferrara, Italy. Neurol Sci .

14. Annegers JF, Hauser WA, Coan SP, Rocca WA (1998) A population-based study of seizures after traumatic brain injuries. N Engl J Med 338: 20-24.

15. Asikainen I, Kaste M, Sarna S (1999) Early and late posttraumatic seizures in traumatic brain injury rehabilitation patients: brain injury factors causing late seizures and influence of seizures on long-term outcome. Epilepsia 40: 584589.

16. Hahn YS, Fuchs S, Flannery AM, Barthel MJ, McLone DG (1988) Factors influencing posttraumatic seizures in children. Neurosurgery 22: 864-867.

17. Annegers JF, Grabow JD, Groover RV, Laws ER Jr, Elveback LR, et al. (1980) Seizures after head trauma: a population study. Neurology 30: 683-689.

18. Rosati G, Granieri E (1990) Manuale di Neuroepidemiologia: Epidemiologia dell'Epilessia. La Nuova Italia Scientifica, Roma: 66-106.

19. Yeh CC, Chen TL, Hu CJ, Chiu WT, Liao CC (2013) Risk of epilepsy after traumatic brain injury: a retrospective population-based cohort study. J Neurol Neurosurg Psychiatry 84: 441-445.

20. Jennett B (1975) William Heinemann Medical Books: Epilepsy after non-missile head injuries. England.

21. Angeleri F, Majkowski J, Cacchiò G, Sobieszek A, D'Acunto S, et al. (1999) Posttraumatic epilepsy risk factors: one-year prospective study after head injury. Epilepsia 40: 1222-1230.

22. Schaumann BA, Annegers JF, Johnson SB, Moore KJ, Lubozynski MF, et al. (1994) Family history of seizures in posttraumatic and alcohol-associated seizure disorders. Epilepsia 35: 48-52.

23. Diaz-Arrastia R, Agostini MA, Frol AB, Mickey B, Fleckenstein J, et al. (2000) Neurophysiologic and neuroradiologic features of intractable epilepsy after traumatic brain injury in adults. Arch Neurol 57: 1611-1616.

24. Mathern GW, Babb TL, Vickrey BG, Melendez M, Pretorius JK (1994) Traumatic compared to non-traumatic clinical-pathologic associations in temporal lobe epilepsy. Epilepsy Res 19: 129-139.

25. Haltiner AM, Temkin NR, Dikmen SS (1997) Risk of seizure recurrence after the first late posttraumatic seizure. Arch Phys Med Rehabil 78: 835-840.

26. Willmore LJ, Sypert GW, Munson JB (1978) Recurrent seizures induced by cortical iron injection: a model of posttraumatic epilepsy. Ann Neurol 4: 329336.

27. Rosen AD, Frumin NV (1979) Focal epileptogenesis after intracortica hemoglobin injection. Exp Neurol 66: 277-284

28. Mukherjee S, Zeitouni S, Cavarsan CF, Shapiro LA (2013) Increased seizure susceptibility in mice 30 days after fluid percussion injury. Front Neurol 4: 28

29. Gennarelli TA, Thibault LE, Adams JH, Graham DI, Thompson CJ, et al. (1982) Diffuse axonal injury and traumatic coma in the primate. Ann Neurol 12: 564 574.

30. Katayama Y, Becker DP, Tamura T, Hovda DA (1990) Massive increases in extracellular potassium and the indiscriminate release of glutamate following concussive brain injury. J Neurosurg 73: 889-900.

31. Tani H, Bandrowski AE, Parada I, Wynn M, Huguenard JR, et al. (2007) Modulation of epileptiform activity by glutamine and system $A$ transport in model of post-traumatic epilepsy. Neurobiol Dis 25: 230-238.

32. McKinney RA, Debanne D, Gähwiler BH, Thompson SM (1997) Lesion-induced axonal sprouting and hyperexcitability in the hippocampus in vitro: implications for the genesis of posttraumatic epilepsy. Nat Med 3: 990-996.

33. Lombroso CT (2000) Can early postnatal closed head injury induce cortica dysplasia. Epilepsia 41: 245-253.

34. Jennett B, Van De Sande J (1975) EEG prediction of post-traumatic epilepsy. Epilepsia 16: 251-256.

35. Heikkinen ER, Rönty HS, Tolonen U, Pyhtinen J (1990) Development of posttraumatic epilepsy. Stereotact Funct Neurosurg 54-55: 25-33.

36. Messori A, Polonara G, Carle F, Gesuita R, Salvolini U (2005) Predicting posttraumatic epilepsy with MRI: prospective longitudinal morphologic study in adults. Epilepsia 46: 1472-1481.

37. Hoff H (1947) Fortschritte in der Behandlung der Epilepsie. Monatssch Psychiatr Neurol 114:105-118

38. Temkin NR, Dikmen SS, Wilensky AJ, Keihm J, Chabal S, et al. (1990) A randomized, double-blind study of phenytoin for the prevention of posttraumatic seizures. N Engl J Med 323: 497-502.

39. Temkin NR (2001) Antiepileptogenesis and seizure prevention trials with antiepileptic drugs: meta-analysis of controlled trials. Epilepsia 42: 515-524.

40. Glötzner FL, Haubitz I, Miltner F, Kapp G, Pflughaupt KW (1983) [Seizure prevention using carbamazepine following severe brain injuries]. Neurochirurgia (Stuttg) 26: 66-79.

41. Temkin NR, Dikmen SS, Anderson GD, Wilensky AJ, Holmes MD, et al. (1999) Valproate therapy for prevention of posttraumatic seizures: a randomized trial J Neurosurg 91:593-600

42. Jones KE, Puccio AM, Harshman KJ, Falcione B, Benedict N, et al. (2008) Levetiracetam versus phenytoin for seizure prophylaxis in severe traumatic brain injury. Neurosurg Focus 25: E3.

43. Klein P, Herr D, Pearl PL, Natale J, Levine Z, et al. (2012) Results of phase 2 safety and feasibility study of treatment with levetiracetam for prevention of posttraumatic epilepsy. Arch Neurol 69: 1290-1295.

44. Mori A, Yokoi I, Noda Y, Willmore LJ (2004) Natural antioxidants may preven posttraumatic epilepsy: a proposal based on experimental animal studies. Acta Med Okayama 58: 111-118

45. Levy SL, Burnham WM, Hwang PA (1990) An evaluation of the anticonvulsant effects of vitamin E. Epilepsy Res 6: 12-17.

46. Yokoi I, Kabuto H, Yamamoto M, Mori A (1990) Effect of ferric ion and active oxygen scavenger on monoamine release in the striatum of the rat. Jpn J Psychiatry Neurol 44: 440-441.

47. Temkin NR, Anderson GD, Winn HR, Ellenbogen RG, Britz GW, et al (2007) Magnesium sulfate for neuroprotection after traumatic brain injury: a randomised controlled trial. Lancet Neurol 6: 29-38.

48. Malhotra J, Gupta YK (1997) Effect of adenosine receptor modulation on pentylenetetrazole-induced seizures in rats. Br J Pharmacol 120: 282-288.

49. Yokoi I, Toma J, Liu J, Kabuto H, Mori A (1995) Adenosines scavenged hydroxyl radicals and prevented posttraumatic epilepsy. Free Radic Biol Med 19: 473-479. 
50. Philo R, Reiter RJ (1978) Characterization of pinealectomy induced convulsions in the Mongolian gerbil (Meriones unguiculatus). Epilepsia 19: 485-492.

51. Srivastava AK, Gupta SK, Jain S, Gupta YK (2002) Effect of melatonin and phenytoin on an intracortical ferric chloride model of posttraumatic seizures in rats. Methods Find Exp Clin Pharmacol 24: 145-149.
52. Myseros JS, Bullock R (1995) The rationale for glutamate antagonists in the treatment of traumatic brain injury. Ann N Y Acad Sci 765: 262-271.

53. Ikonomidou C, Turski L (2002) Why did NMDA receptor antagonists fail clinical trials for stroke and traumatic brain injury? Lancet Neurol 1: 383-386. 